\title{
Kernos
}

Revue internationale et pluridisciplinaire de religion grecque antique

\section{2| 1989}

Varia

\section{Des nouvelles de « Mentor »}

Inauguration d'une banque de données bibliographiques sur la religion grecque antique

\section{Vinciane Pirenne-Delforge}

\section{(2) OpenEdition \\ Journals}

Édition électronique

URL : http://journals.openedition.org/kernos/275

DOI : 10.4000/kernos. 275

ISSN : 2034-7871

\section{Éditeur}

Centre international d'étude de la religion grecque antique

Édition imprimée

Date de publication : 1 janvier 1989

Pagination : 283-284

ISSN : 0776-3824

Référence électronique

Vinciane Pirenne-Delforge, « Des nouvelles de « Mentor » », Kernos [En ligne], 2 | 1989, mis en ligne le 04 mars 2011, consulté le 06 mai 2019. URL : http://journals.openedition.org/kernos/275 


\title{
DES NOUVELLES DE «MENTOR». INAUGURATION D'UNE BANQUE DE DONNÉES BIBLIOGRAPHIQUES SUR LA RELIGION GRECQUE ANTIQUE
}

\author{
par Vinciane PIRENNE-DELFORGE
}

L'année dernière, dans les pages de cette revue, nous avions présenté le programme de bibliographie critique de la religion grecque antique en cours de réalisation à l'Université de Liège sous le nom évocateur de "Mentor» 1 . Sous le titre, toujours embarrassant, de «Délais de réalisation», l'inauguration de la banque de données était annoncée pour le début de l'année 1989. Et, en effet, dans le cadre du colloque "Oracles et mantique dans la religion grecque ancienne» (Liège, les 16, 17 et 18 mars 1989) aura lieu la mise en service annoncée.

Les références disponibles couvrent principalement la période 1945-1985 (environ 4500 publications). Les trois quarts de cette matière mettent déjà à la disposition des interrogateurs un résumé accompagné d'une appréciation critique pour chaque publication. Dans le souci d'offrir le maximum d'informations dès à présent, les articles et ouvrages dont le résumé critique ne nous a pas encore été renvoyé par le collaborateur qui en a la charge seront néanmoins intégrés à la banque, accompagnés de la mention "En cours» et indexés sommairement d'après le titre.

En ce qui concerne la période antérieure à 1945 , le dépouillement des références est déjà largement entamé, et l'analyse critique des publications vient de commencer. Mais afin de rendre service aux utilisateurs sans attendre la fin de cette partie du programme, ces références seront disponibles sur base d'une indexation sommaire et sans commentaire critique.

Le guide bibliographique sera quant à lui publié dans le courant de l'année 1990 et couvrira, de manière sélective, la production scientifique antérieure à 1986 qui présente encore un intérêt pour le chercheur d'aujourd'hui. Le premier supplément au guide couvrira selon toute vraisemblance la période 1986-1990.

1 Voir Kernos, 1 (1988), p. 273-276. 


\title{
Comment interroger la banque de données?
}

«Mentor» n'étant pas encore intégré dans les réseaux internationaux, il est indispensable de passer par l'intermédiaire des promoteurs du programme pour procéder à une interrogation. Trois possibilités s'offrent au chercheur désireux d'obtenir une information : le courrier, le téléphone, la visite personnelle.

Pour formuler adéquatement la (les) question(s), il convient de se reporter au guide d'interrogation (provisoire) qui est dès à présent disponible à l'adresse reprise ci-dessous, au prix de $200 \mathrm{FB}$. Ce guide comprend, outre une introduction sur les objectifs généraux de "Mentor» et la liste des collaborateurs, une présentation de l'Organon, c'est-à-dire un ensemble de rubriques correspondant aux principaux aspects de la religion grecque (voir Kernos, 1 [1988], p. 275), le Thesaurus, c'est-à-dire la liste alphabétique des descripteurs accessibles dans l'état actuel de la banque, ainsi que quelques modèles d'interrogation. On y trouvera en outre les informations concernant le coût de l'interrogation et les modalités de paiement.

Pour tout renseignement complémentaire et pour l'interrogation de la banque, s'adresser à

\author{
André MotTe ou Paul Wathelet \\ ouVinciane PIRENNE-DELFORGE \\ Université de Liège \\ Place du XX-Août 32 \\ $B$ - 4000 Liège \\ Belgique
}

Tél. : (0)41/42.00.80 poste 597 ou 581 ou 568 